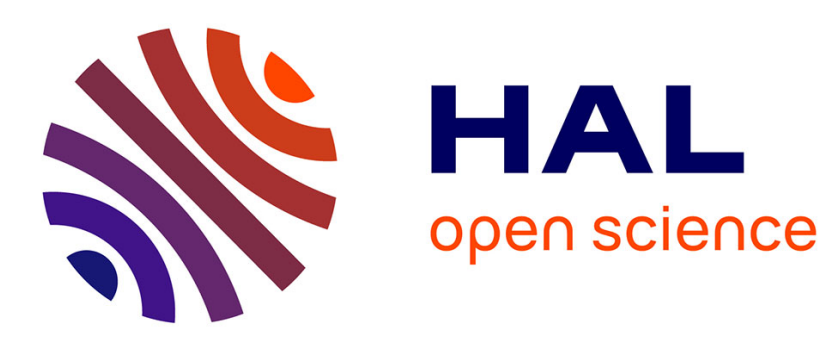

\title{
EVM measurement of RF ZigBee transceivers using standard digital ATE
}

Thibault Vayssade, Florence Azaïs, Laurent Latorre, François Lefèvre

\section{To cite this version:}

Thibault Vayssade, Florence Azaïs, Laurent Latorre, François Lefèvre. EVM measurement of RF ZigBee transceivers using standard digital ATE. DFT 2020 - 33rd IEEE International Symposium on Defect and Fault Tolerance in VLSI and Nanotechnology Systems, Oct 2020, Frascati, Italy. pp.1-6, 10.1109/DFT50435.2020.9250900 . lirmm-03000882

\section{HAL Id: lirmm-03000882 https://hal-lirmm.ccsd.cnrs.fr/lirmm-03000882}

Submitted on 12 Nov 2020

HAL is a multi-disciplinary open access archive for the deposit and dissemination of scientific research documents, whether they are published or not. The documents may come from teaching and research institutions in France or abroad, or from public or private research centers.
L'archive ouverte pluridisciplinaire HAL, est destinée au dépôt et à la diffusion de documents scientifiques de niveau recherche, publiés ou non, émanant des établissements d'enseignement et de recherche français ou étrangers, des laboratoires publics ou privés. 


\title{
EVM measurement of RF ZigBee transceivers using standard digital ATE
}

\author{
T. Vayssade ${ }^{(1,2)}$, F. Azaïs ${ }^{(1)}$, L. Latorre ${ }^{(1)}$, F. Lefevre ${ }^{(2)}$ \\ (1) LIRMM, Univ. Montpellier, CNRS, 161 rue Ada, Montpellier, France \\ (2) NXP Semiconductors, 2 Espl. Anton Phillips, 14000 Caen, France
}

\begin{abstract}
This paper targets the challenging issue of production test cost reduction for RF circuits. More specifically, it proposes a low-cost solution to perform EVM measurement of ZigBee transceivers using only a standard digital test equipment. The approach is based on 1-bit under-sampled acquisition of the RF modulated-signal by a digital tester channel associated with a specifically-tailored processing algorithm. The different steps of the post-processing algorithm are detailed in the paper. Hardware experimental results obtained on both a Universal Software Radio Peripheral (USRP) that emulates the circuit-under-test and on an actual ZigBee transceiver IC are presented.
\end{abstract}

Keywords - RF test; digital ATE; EVM measurement; digital signal processing; wireless communication; ZigBee; OQPSK

\section{INTRODUCTION}

Reduction of production testing costs of RF devices is a major issue for semiconductor manufacturers. Indeed, with the booming of the Internet-of-Things (IoT), these devices are today used in an increasing number of applications and they are produced in very high volumes. The current industrial test practice relies on the use of an ATE equipped with expensive RF instruments that realize various measurements of the device performance such as power level, spectral leakage, or Error Vector Magnitude (EVM).

An interesting approach to lower the testing costs is to develop alternative solutions that can be applied on digital ATE. Recent works following this approach and targeting RF devices can be found in the literature, e.g. based on the use of a reference transceiver accompanied by a FPGA to handle the interface between the reference transceiver and the digital ATE [1], based on the use of a processor embedded in a radio SoC that implements a self-test and delivers low-speed digital signals processed by the digital ATE [2], or defining a digital ATE system with multi-level drivers and comparators to perform direct modulation/demodulation of QAM signals [3]. Another strategy is based on a direct acquisition of the analog/RF signal with a standard digital tester channel and the development of dedicated post-processing algorithms able to retrieve the essential signal characteristics from the binary capture. This strategy has been exploited for signals in the intermediate frequency range (few tens of $\mathrm{MHz}$ ) targeting the demodulation of basic FM/AM schemes [4] or phase noise characterization [5].

This paper targets the development of a low-cost solution for EVM measurement of ZigBee transceivers based on a direct acquisition of the RF signal with a standard ATE channel. The challenge is here that we deal with a high frequency signal at $2.4 \mathrm{GHz}$ and an elaborated modulation scheme, i.e. OQPSK with half-sine pulse shaping.

\section{BACKGROUND}

\section{A. Signal Under Test Characteristics (ZigBee)}

This section briefly summarizes the main characteristics of the signal to be analyzed, which are specified by the IEEE Std $802.15 .4^{\mathrm{TM}}$. In this work, we focus on the $2.4 \mathrm{GHz}$ band, which is an ISM band accepted worldwide.

The modulation format is Offset Quadrature Phase Shift Keying (OQPSK) with half-sine pulse shaping. OQPSK is a variant of QPSK where an offset of one-bit period (half-symbol period) is added on the signal in the $\mathrm{Q}$ branch in order to ensure that signals in the I and Q branches never change at the same time, which limits the phase jump to $90^{\circ}$ and reduces amplitude variations. Moreover, half sine pulse shaping is applied in each branch after the classical NRZ encoding in order to smooth the phase transitions and ensure a constant modulation envelope. These two features permit to obtain a better spectral efficiency than the classical QPSK modulation format. Finally, another feature specified by the standard is the use of Direct Sequence Spread Spectrum (DSSS) technique in order to reduce the overall signal interference. As a consequence, the chip rate is much higher than the input data rate, i.e. $2 \mathrm{Mchip} / \mathrm{s}$ at the input of the modulator while only $250 \mathrm{~kb} / \mathrm{s}$ for the input data rate.

\section{B. Current test practice}

The current industrial practice for production testing of RF devices relies on the use of an ATE equipped with RF tester channels. Such channels comprise specific hardware resources (mixer, filters, ADC) that realize the acquisition of an RF signal and convert it into a digitized version in the Intermediate Frequency (IF) band; software DSP procedures are then applied on the digitized data to implement various measurements (power level, spectral leakage, EVM...).

The main drawback of this solution is its cost. Indeed, because of the required hardware resources with very exigent performances, the cost of a RF tester channel is extremely high and constitutes a major contributor to the cost of testing RF devices.

\section{Error vector magnitude (EVM)}

Error vector magnitude (EVM) is the most widely used metric to express the modulation quality in wireless communication systems. Basically, it is a measure of the signal constellation deviation from its ideal reference. It is computed from error vectors defined in the I-Q plane at the instants in time when symbols are detected. As illustrated in Figure 1, the error vector is the vector difference at a given time between the actual symbol location and the ideal one. EVM is then defined as the as the Root Mean Square (RMS) amplitude of the error vector 
over a sequence of emitted symbols, normalized to ideal signal amplitude reference.

$$
E V M=\frac{\sqrt{\frac{1}{N} \sum_{i=1}^{N}\left(\delta I_{i}{ }^{2}+\delta Q_{i}{ }^{2}\right)}}{S}
$$

The ideal signal amplitude reference $S$ can either be the average symbol amplitude or the maximum symbol amplitude. In case of ZigBee products, IEEE Std 802.15.4 ${ }^{\mathrm{TM}}$ specifies normalization with the maximum signal amplitude.

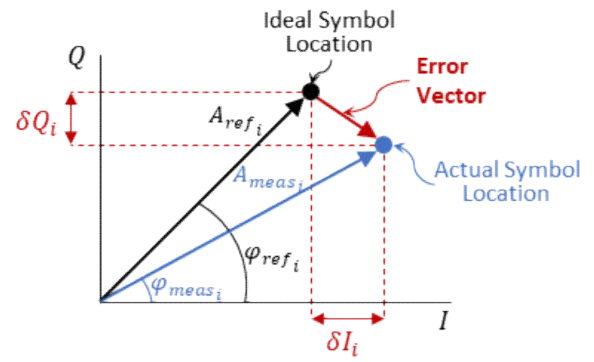

Fig.1: Error vector magnitude (EVM)

\section{PROPOSED DIGITAL TEST SOLUTION}

Our strategy to reduce RF testing costs is to propose new solutions that can be applied using low-cost standard digital tester channels instead of expensive RF tester channels. As illustrated in Figure 2, the basic principle is to perform 1-bit acquisition of the RF signal using the comparator and the latch comprised in a digital tester channel and then to apply a dedicated processing algorithm able to retrieve the RF signal characteristics from the captured binary vector. Key challenges are (i) to determine appropriate conditions for the digital capture that permit to preserve the essential information contained in the $\mathrm{RF}$ signal and (ii) to define the dedicated processing algorithm able to retrieve the desired signal characteristics.

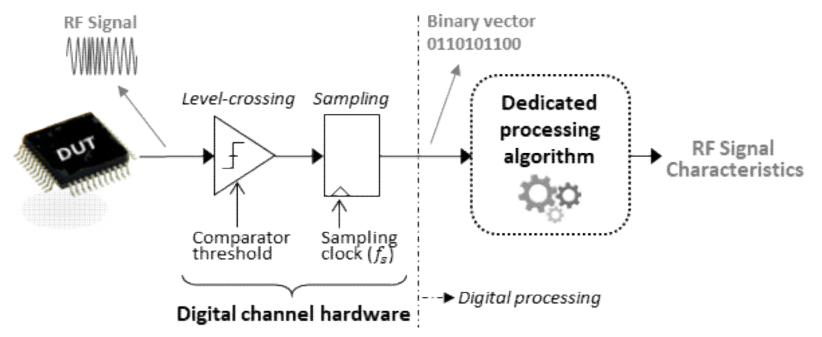

Fig.2: Basic principle of the proposed approach

Regarding the digital capture, the fundamental concept is to under-sample the RF modulated-signal with a sampling rate $f_{s}$ close to a sub-multiple of its carrier frequency $f_{c}$ :

$$
f_{s}=f_{c} / n+f_{d}
$$

where $n$ is the sub-multiple of the carrier frequency and $f_{d}$ is the frequency deviation between the sampling frequency and the carrier frequency sub-multiple.

This under-sampling process produces a digital signal with a fundamental beat frequency $f_{b}=\left|n f_{s}-f_{c}\right|=n\left|f_{d}\right|$ at a much lower range, but that still contains relevant information about the original RF signal. In our case, $n=2$ is sufficient to comply with the maximum sampling rate of standard digital tester channel (typically 1.6Gbps). The same principle (without the sub-multiple) has been exploited in [6,7] to perform precise onchip timing measurements on high speed digital signals.
The aim of the post-processing algorithm is then to retrieve the appropriate information contained the low-frequency digital signal captured by the ATE that permits to evaluate the desired RF performance. Solutions have already been developed in previous works for symbol error detection [8], and power and spectral measurements [9]. In this work, we further extend the approach by developing an original algorithm that permits to realize EVM measurements.

A global overview of the post-processing algorithm is given in Figure 3. It involves three main steps. The first one is dedicated to the processing of the binary discrete-time vector captured by the ATE to extract relevant information regarding amplitude and phase fluctuation of the RF signal and convert this information into analog continuous-time waveforms. This information is then used in the second step in two parallel branches dedicated to (i) reference data generation and (ii) measurement data preparation. Note there are interactions between these two branches, for instance regarding correction of the extracted phase fluctuation and determination of the sampling instants for both measured and reference data. Finally, the last step of the algorithm simply consists in the EVM calculation from measured and reference constellations. These different steps are detailed in the following section.

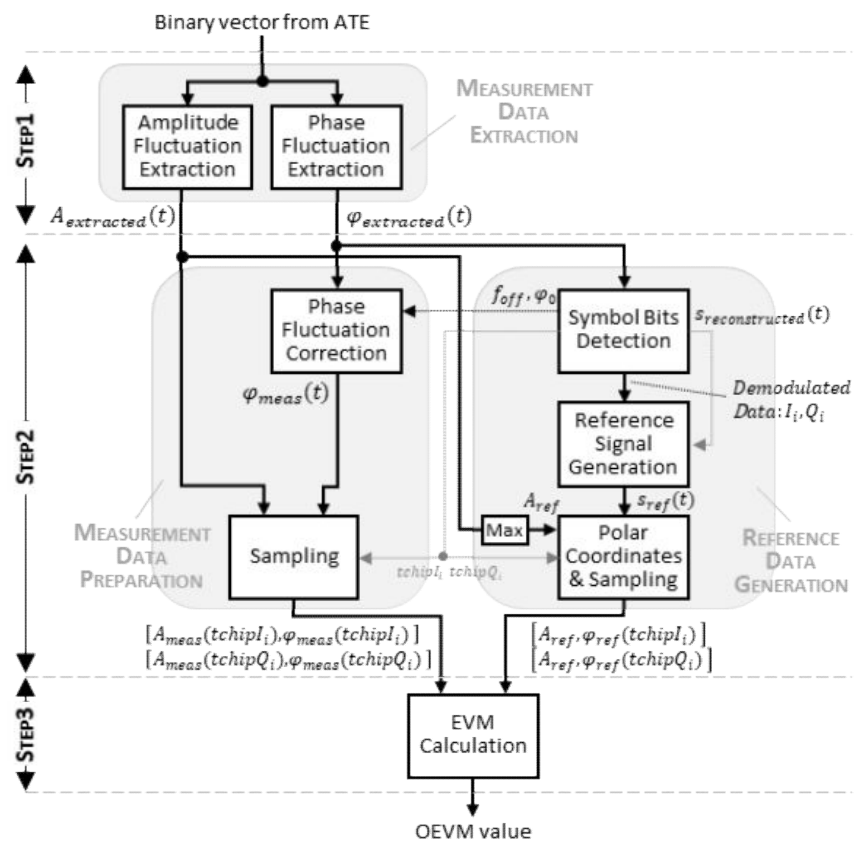

Fig.3: Block diagram of the post-processing algorithm

\section{Post-Processing AlgorithM}

\section{A. Step 1: Measurement data extraction}

The first step of the algorithm consists in processing the binary vector captured by the ATE in order to obtain analog continuous-time estimation of both the phase and amplitude fluctuations of the RF signal. These two estimations can be realized in parallel.

\section{1) Phase fluctuation extraction}

A first task of the algorithm is to retrieve the phase variation of the RF modulated signal. This is an essential task since all the information that has to be delivered by a ZigBee transmitter is 
encoded in the phase variation of the RF modulated signal. The implemented solution relies on the work developed in [8] for symbol error detection using digital test resources. It involves several operations as depicted in Figure 4, which are briefly summarized hereafter.

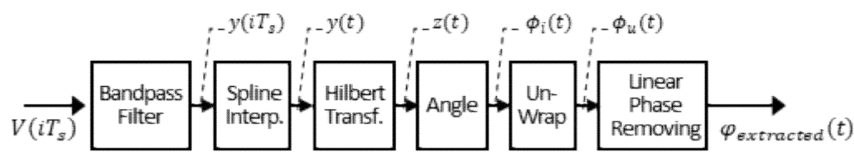

Fig.4: Phase fluctuation extraction from the binary vector captured by the ATE

The digital signal $V\left(i T_{s}\right)$ captured by the ATE is first filtered by band-pass filter centered on the square-wave beat frequency (forward-backward filtering is used to implement a zero-phase filter), which eliminates harmonics introduced by 1-bit quantization and generate a discrete-time sine-wave $y\left(i T_{s}\right)$. Spline interpolation followed by Hilbert Transform are then applied to obtain a time-continuous expression of the signal in the complex plane $z(t)$. The instantaneous phase $\Phi_{i}(t)$ is then computed by taking the angle of the complex signal $z(t)$ with $\Phi_{i}(t)=\operatorname{atan}(\operatorname{Im}(z(t) / \operatorname{Re}(z(t))$. This computation leads to a wrapped phase, which needs to be unwrapped to obtain the linear evolution of the instantaneous phase $\Phi_{u}(t)$, which can be expressed as:

$$
\Phi_{u}(t)=2 \pi f_{b} t+\varphi(t)
$$

where the $1^{\text {st }}$ term corresponds to the linear phase of the squarewave signal with a beat frequency $f_{b}$ and the $2^{\text {nd }}$ term to the phase fluctuation $\varphi(t)$ of the modulated RF signal.

Finally subtracting the linear phase and using the expression of the fundamental beat frequency, an estimation $\varphi_{\text {extracted }}(t)$ of the RF signal phase fluctuation is given by:

$$
\varphi_{\text {extracted }}(t)=\Phi_{u}(t)-2 \pi\left|2 f_{s}-f_{c}\right| t
$$

Note that this expression of the RF signal phase fluctuation has been established under ideal conditions, i.e. assuming that the carrier frequency is exactly known and that there is a perfect synchronization between the ATE and the DUT. Clearly, such conditions are impossible to achieve in a practical environment and it most likely that imperfections will be present in the extracted phase fluctuation, which can be expressed by:

$$
\varphi_{\text {extracted }}(t)=\varphi(t)+2 \pi f_{\text {off }} t+\varphi_{0}
$$

where $\varphi(t)$ is the actual phase fluctuation of the signal under test, $f_{\text {off }}$ is the frequency difference between the assumed carrier frequency and the actual one, and $\varphi_{0}$ is an unknown phase offset related to imperfect or absent synchronization.

These imperfections need to be corrected to ensure accurate EVM measurement; corrections will be performed in the second step of the processing algorithm.

\section{2) Amplitude fluctuation extraction}

In parallel of phase fluctuation extraction, a second task is to retrieve the low-frequency amplitude variations that might affect the RF signal envelope. Theoretically an OQPSK-modulated signal with half sine pulse shaping exhibits a constant envelope. However, imperfections in the modulator such as IQ imbalance or power drop can lead to low-frequency amplitude variations. Such variations must be taken into account to obtain accurate EVM measurement.
The implemented solution utilizes the work developed in [9] for power measurement using digital test resources. The fundamental basis comes from the principle of level-crossing that converts voltage-domain information into timing information. Indeed, assuming an ideal sine-wave converted into a square-wave by level-crossing (with a comparator threshold $C$ different than 0 ), there is a direct relationship between the sinewave amplitude $A$ and the square-wave duty cycle $\alpha$ :

$$
A=\frac{C}{\cos (\pi \alpha)}
$$

In our context, this principle can be exploited to estimate low-frequency amplitude variations of the RF signal by monitoring the duty cycle of the digital signal captured by the ATE. Indeed, this digital signal corresponds to a square-ware signal generated by a level-crossing operation combined with an under-sampling process. The under-sampling process translates the square-wave signal towards a lower frequency but preserves its essential characteristics, in a limited bandwidth. The variations of the digital signal duty cycle therefore give an image of the variations of the RF signal envelope.

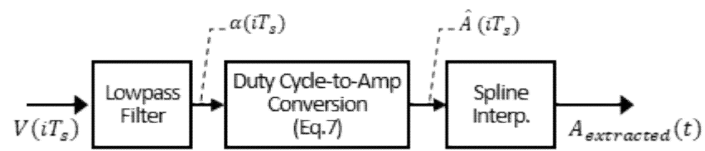

Fig.5: Amplitude fluctuation extraction from the binary vector captured by the ATE

Practically, the implemented solution involves three consecutive operations, as illustrated in Figure 5. The first one consists in measuring the digital signal duty cycle $\alpha$ by applying a low-pass filter on the captured digital signal $V\left(i T_{s}\right)$. In this work, we have chosen a type II Chebyshev filter with stopband starting at $7 \mathrm{MHz}$. The filter has been designed to pass the components related to low-frequency envelope variations (i.e. with a bandwidth greater than the chip rate of $1 \mathrm{MHz}$ ), but eliminate all components related to the square waveform of the digital signal (i.e. with a cut-off frequency lower than the fundamental beat frequency of $20 \mathrm{MHz}$ ). Here again, forwardbackward filtering is used to implement a zero-phase filter. The output of the filter is a discrete-time signal $\alpha\left(i T_{S}\right)$ whose value is representative of the digital signal duty cycle. Duty cycle-toamplitude conversion is then simply realized based on Eq.(6):

$$
\hat{A}\left(i T_{S}\right)=\frac{C}{\cos \left(\pi \alpha\left(i T_{S}\right)\right)}
$$

Finally, spline interpolation is applied to obtain a continuous-time signal $A_{\text {extracted }}(t)$ that matches with the time resolution of the extracted phase. This signal corresponds to an estimation of the RF signal amplitude fluctuation.

\section{B. Step 2: Measurement data preparation and reference data generation}

The second step of the algorithm is dedicated to the preparation of measurement data and the generation of reference data. These operations are realized in parallel, but with some interactions regarding correction of the extracted phase fluctuation and timing alignment of measured and reference data.

\section{1) Reference data generation}

The first task of the reference data generation process is to recover the symbols bits that have been delivered by the DUT. 
The solution that has been implemented relies on a three-step process as illustrated in Figure 6.

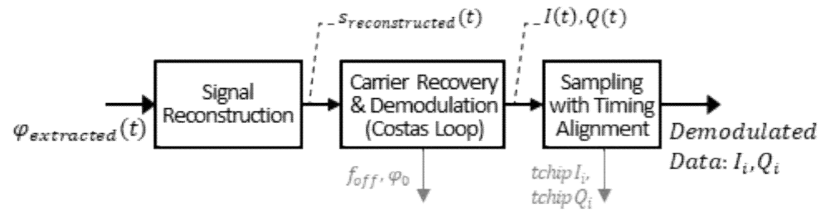

Fig.6: Detection of symbol bits from extracted phase fluctuation

The first step consists in performing the reconstruction in phase of the RF modulated signal emitted by the DUT by adding the extracted phase fluctuation to the ideal instantaneous linear phase of the carrier:

$$
s_{\text {reconstructed }}(t)=\sin \left(2 \pi f_{c} t \pm \varphi_{\text {extracted }}(t)\right)
$$

Note that depending whether the sampling frequency is chosen with a positive or negative deviation with respect to the carrier half-frequency, the extracted phase fluctuation should be added or subtracted to take into account spectral inversion.

The second step is dedicated to carrier recovery as well as demodulation. This is accomplished through the use of a Costas loop. A Costas loop is a PLL-based circuit that uses coherent quadrature signals to measure a phase error, which in turn is used to regulate the loop oscillator. When locked, quadrature signals correspond to the demodulated data.

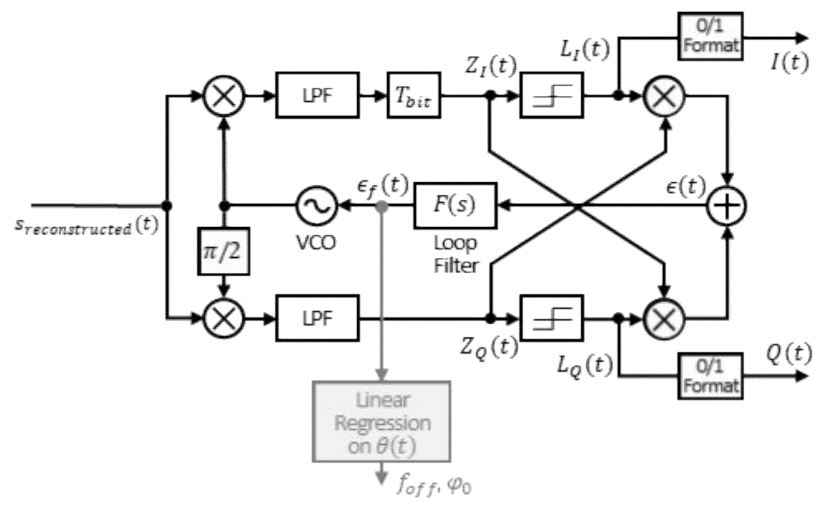

Fig.7: Costas loop for demodulation of OQPSK signals with pulse shaping

In this work, we use a software model of the Costas loop modified to manage OQPSK with half sine pulse shaping. Its architecture is given in Figure 7. The input of the loop is the reconstructed RF signal, which is split in two $I$ and $Q$ branches. Signals on the $I$ and $Q$ branches are respectively multiplied by a VCO-generated tone and a $90^{\circ}$ shifted version of this tone; low pass filtering is then applied to remove the double frequency component and perform the demodulation. By taking the difference between the absolute value of the demodulated $I$ branch and $Q$ branch, an error is obtained; this error $\varepsilon(t)$ passes through a loop filter and drives the VCO to achieve phase and frequency locking. Note that in the specific context of OQPSK with half sine pulse shaping, a delay of one-bit period $\left(T_{b i t}\right)$ is added on the $I$ branch to compensate for the delay that has been added on the $Q$ branch during the modulation process. Finally in the locked state, $Z_{I}(t)$ and $Z_{Q}(t)$ correspond to reconstructed waveforms of the original quadrature signals generated within the DUT and $I(t)$ and $Q(t)$ are the binary representation of these signals.
Another interest of the Costas loop is that the frequency and phase offsets present in the signal captured by the ATE can be evaluated from a simple processing of its error signal $\varepsilon(t)$. More precisely, the phase correction $\Theta(t)$ performed by the loop can be computed by integrating the filtered error signal $\varepsilon_{f}(t)$ with:

$$
\Theta(t)=\int_{0}^{t} 2 \pi \varepsilon_{f}(t) \cdot d t
$$

As illustrated in Figure 8, this signal exhibits a linear trend that can be fitted with a function of the form $a * t+b$. The slope coefficient depends on the difference between the actual carrier frequency and the initial VCO frequency with $a=2 \pi\left(f_{c_{\text {meas }}}-\right.$ $\left.f_{c_{\text {coarse }}}\right)$, while the intercept term directly corresponds to the initial phase offset $\varphi_{0}$. Consequently, we can estimate the frequency and phase offsets present in the signal captured by the ATE with:

$$
\begin{aligned}
& f_{o f f}=f_{c}-\left(f_{c_{\text {coarse }}}+\frac{a}{2 \pi}\right) \\
& \varphi_{0}=b
\end{aligned}
$$

where $f_{c_{\text {meas }}}$ is the measured value of the actual carrier frequency.

These values will the used to correct the extracted phase fluctuation $\varphi_{\text {extracted }}(t)$ in the branch dedicated to measurement data preparation.

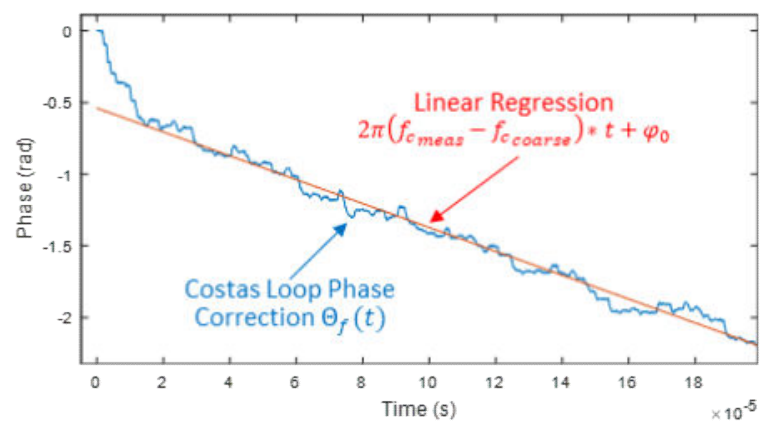

Fig.8: Linear regression on Costas loop phase correction $\boldsymbol{\Theta}(\boldsymbol{t})$

Finally, the last step of symbol bits detection simply consists in sampling the reconstructed $I(t)$ and $Q(t)$ binary signals with a $1 / 2 T_{\text {bit }}$ clock frequency. Note that to ensure correct interpretation, the sampling should occur in the middle of the period of each binary signal, which means that a specific timing alignment has to be defined for each binary signal. A dedicated procedure has been developed which determines appropriate tchipI and tchipQ sampling instants. These instants will be used later in the algorithm to sample both the measured and reference data.

The following task is to generate a RF reference signal $s_{\text {ref }}(t)$ using the demodulated data. For this, the demodulated data are fed into an ideal modulator model centered on the measured carrier frequency $f_{c_{\text {meas }}}$. The output of the modulator is then filtered by a band-pass filter to consider the limited bandwidth of the measurement instrument and synchronized with the reconstructed RF signal $s_{\text {reconstructed }}(t)$.

Finally, the last task is to determine the polar coordinates of the reference data. The radial coordinate is constant whatever the emitted symbol since it is a specific feature of OQPSK with halfsine pulse shaping to have a constant modulation envelope. Its value depends on the amplitude level of the signal emitted by the 
DUT. It is computed by taking the maximum value of the extracted RF signal amplitude fluctuation:

$$
A_{\text {ref }}=\max \left[A_{\text {extracted }}(t)\right]
$$

The angular coordinate corresponds to the phase of the reference signal $s_{\text {ref }}(t)$; it is computed by taking the angle of the complex representation obtained from Hilbert transform. This reference phase $\varphi_{r e f}(t)$ is then simply sampled at the tchipI and tchipQ instants determined in the first task.

\section{2) Measurement data preparation}

The main task of the measurement data preparation is to correct the extracted phase fluctuation $\varphi_{\text {extracted }}(t)$, taking into account the frequency offset $f_{\text {off }}$ and the phase offset $\varphi_{0}$ determined by the Costas loop in the reference data generation process. This is done by subtracting the supplementary linear phase evolution introduced by the frequency offset and the measure phase offset to the original extracted phase fluctuation:

$$
\varphi_{\text {meas }}(t)=\varphi_{\text {extracted }}(t)-2 \pi f_{\text {off }} t-\varphi_{0}
$$

Finally, this corrected phase fluctuation is then simply sampled at the tchipI and tchipQ instants, as well the extracted amplitude fluctuation to produce the measurement data.

\section{Step 3: EVM calculation}

This last step finalizes the process by implementing the EVM calculation. It takes as inputs the measured and reference data. Note that in case of OQPSK modulation format, the computation uses two points-per-symbols due to the one-bit period offset between I and Q data. In this case, the computed value is called Offset Error Vector Magnitude (OEVM). Practically for each decoded symbol, the errors $\delta I_{i}$ and $\delta Q_{i}$ are respectively computed at the $\operatorname{tchip} I_{i}$ and $\operatorname{tchip} Q_{i}$ instants with:

$$
\begin{aligned}
\delta I_{i}= & A_{\text {meas }}\left(\operatorname{tchip} I_{i}\right) * \cos \left(\varphi_{\text {meas }}\left(\operatorname{tchip}_{i}\right)\right) \\
& -A_{\text {ref }} * \cos \left(\varphi_{\text {ref }}\left(t \operatorname{tchip} I_{i}\right)\right) \\
\delta Q_{i}= & A_{\text {meas }}\left(\operatorname{tchip} Q_{i}\right) * \cos \left(\varphi_{\text {meas }}\left(\operatorname{tchip}_{i}\right)\right) \\
& -A_{\text {ref }} * \cos \left(\varphi_{\text {ref }}\left(\operatorname{tchip} Q_{i}\right)\right)
\end{aligned}
$$

OEVM is then computed as the root mean square of these errors and expressed as a percentage of the reference signal amplitude:

$$
\operatorname{OEVM}(\%)=\frac{\sqrt{\frac{1}{n} \sum_{i=1}^{n}\left(\delta I_{i}{ }^{2}+\delta Q_{i}{ }^{2}\right)}}{A_{\text {ref }}} * 100
$$

\section{VALIDATION}

\section{A. Experimental setup}

The experimental setup depicted in Figure 9 has been developed to evaluate the proposed solution. In a first version, a Universal Software Defined Radio Peripheral (USRP) is used to emulate the Device Under Test (DUT). It is programmed to deliver a RF signal with a carrier frequency $f_{c}=2.48 \mathrm{GHz}$ (channel 26 as stated in IEEE Std 802.15.4 ${ }^{\mathrm{TM}}$ ), using OQPSK modulation format with half-sine pulse shaping. A key interest in using a USRP is that it is possible to add controlled imperfections such as IQ imbalance or phase noise that degrade the quality of the generated signal and therefore allows the evaluation of the proposed solution over a relatively large range of EVM values. On the other hand, because of its versatility, such product is obviously less performing than a dedicated IC and does not permit to generate a signal with EVM values below $1 \%$. The USRP is therefore replaced by an NXP ZigBee transceiver in a second version, which presents a typical EVM value less than $1 \%$. This IC is also programmed to deliver a RF modulated signal with a carrier frequency $f_{c}=2.48 \mathrm{GHz}$.

The generated RF signal is split in two signals using a resistive power divider. The first one is directly connected to a digital storage oscilloscope and its acquisition is performed at 5 GSamples/s, which satisfies the Nyquist rate. The second one is first sent to a latched comparator (ADCMP 572 from Analog Devices) that emulates the digital tester channel by converting the RF modulated signal into a square-wave signal. The comparator threshold is set at $70 \%$ of the nominal RF signal amplitude and the sampling frequency at $f_{s}=1.25 \mathrm{GHz}$; the output of the latched comparator is therefore a binary signal with a fundamental beat frequency $f_{b}=20 \mathrm{MHz}$. This output is connected to the second channel of the oscilloscope, which performs its acquisition. Both signals captured by the oscilloscope are then transferred on a PC to be further processed. The complete acquisition is conserved for the analysis of the RF signal, while decimation by a factor 4 is applied on the binary signal to keep only $1.25 \mathrm{GSamples} / \mathrm{s}$ in order to match with the latched comparator sampling frequency. Note that this setup does not provide the best experimental conditions because there is no synchronization between the sampling clock of the latched comparator and the oscilloscope. Finally, EVM measurement algorithm is applied on the original RF signal and on the one reconstructed from the binary acquisition.

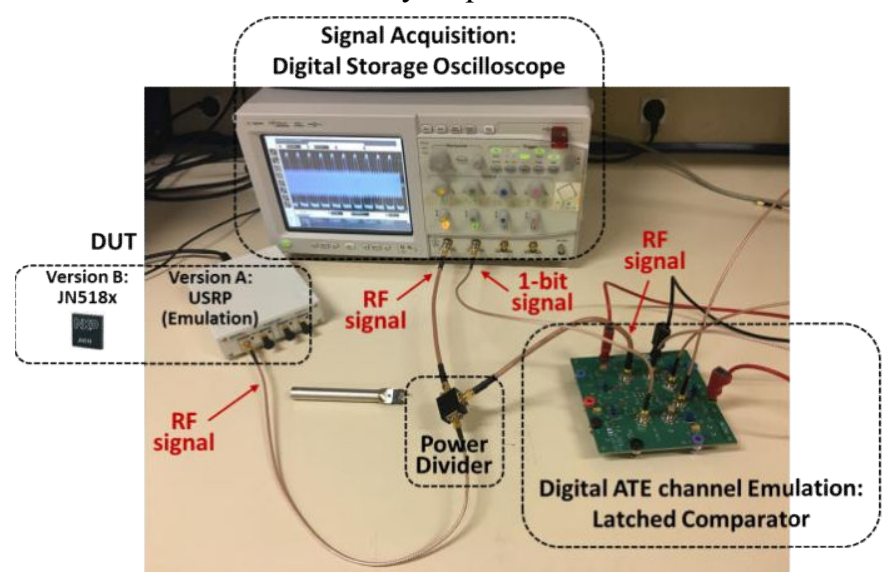

Fig.9: Lab experimental setup

\section{B. Results with DUT emulation (NI USRP)}

First experiments have been performed using the USRP as DUT emulation. Initial measurements have been realized on the signal generated by the USRP without adding any impairment, which gives the floor OEVM of the device. Then additional measurements have been realized under degraded conditions, i.e. with different levels of phase noise injected on the reference VCO of the modulator and with an IQ imbalance of 5\%. Results are summarized in Figure 10. A good agreement is observed between measurements realized on the RF signal and the ones derived from the 1-bit signal, on a large range of EVM values between $1 \%$ to $7 \%$. The difference between measured values is inferior to $0.1 \%$ for EVM below $4 \%$ and slightly increases for higher EVM. The maximum difference is observed in case of 
added IQ imbalance but the error remains lower than $0.5 \%$ for a measured EVM around 6\%. Overall, the average error is only of $-0.03 \%$ and the RMS error of $0.19 \%$. These results clearly demonstrate the potential of the proposed solution to perform EVM measurements based only on a digital acquisition.

\begin{tabular}{|l|c|c|c|c|c|c|}
\multicolumn{7}{c}{ Phase noise } \\
\begin{tabular}{|l|l|l|l|l|} 
OEVM from \\
RF capture
\end{tabular} & $1.20 \%$ & $1.25 \%$ & $1.65 \%$ & $3.30 \%$ & $6.20 \%$ & $5.89 \%$ \\
\hline $\begin{array}{l}\text { OEVM from } \\
\text { 1-bit capture }\end{array}$ & $1.25 \%$ & $1.34 \%$ & $1.68 \%$ & $3.37 \%$ & $6.34 \%$ & $5.45 \%$ \\
\hline
\end{tabular}

Fig.10: RF and 1-bit OEVM measurements on USRP device

\section{Results on actual IC (NXP JN518x ZigBee transceiver)}

Further experiments have been performed replacing the USRP by a real ZigBee transceiver from NXP Semiconductors. Under nominal setting, this device delivers an RF modulatedsignal with a better OEVM than the USRP, i.e. well below $1 \%$. The quality of the signal can be slightly degraded through the programing of an internal configuration register (two possible degraded settings).

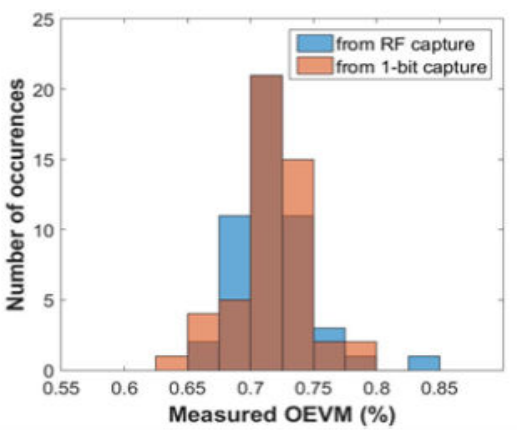

(a) Nominal setting

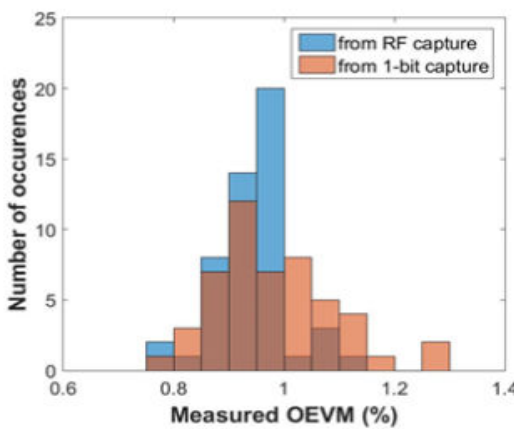

(b) Degraded setting \#1
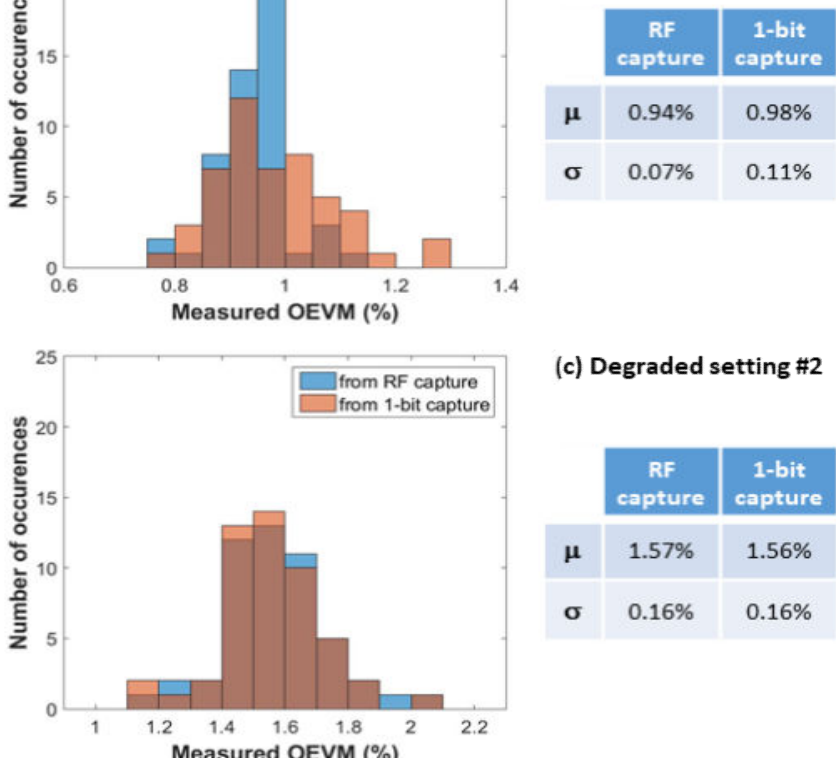

(c) Degraded setting \#2

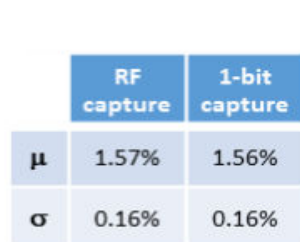

Fig. 11: Histograms of measured OEVM values for different settings of JN518x IC

For each setting, the parallel acquisition of the RF signal and the binary signal delivered by the latched comparator has been realized and OEVM values have been determined. Moreover, in order to study the repeatability of the measurement, this process has been repeated 50 times. Results are illustrated in Figure 11 which shows the histograms of measured OEVM values together with the measurement statistics. A very good agreement can be observed between measurements realized on the RF signal and the ones derived from the 1-bit signal. The difference between the mean OEVM value determined on the RF signal and the one computed on the 1-bit signal does not exceed $0.04 \%$, over the three different settings. Moreover, both solutions lead to a similar dispersion, indicating that there is no degradation of the measurement repeatability by exploiting the binary signal. These results therefore fully validate the ability of the proposed solution to perform accurate EVM measurement based only on a digital acquisition, even in case of low EVM values.

\section{CONCLUSION}

This paper has presented a low-cost digital test solution for EVM measurement of ZigBee transceivers. The technique relies on the use of a standard digital ATE channel to perform undersampled 1-bit acquisition of the RF modulated-signal at a sampling rate close to half the carrier frequency. A dedicated post-processing algorithm has been developed that permits to retrieve the essential RF signal characteristics from the captured binary data and determine the EVM performance. The technique has been validated through hardware measurements on a USRP device and on an actual ZigBee transceiver. Future work will address the validation of the proposed solution on an industrial ATE and the optimization of the post-processing algorithm for efficient integration on the production test floor.

\section{ACKNOWLEDGMENT}

This work has been carried out under the framework of PENTA-EUREKA project "HADES: Hierarchy-Aware and secure embedded test infrastructure for Dependability and performance Enhancement of integrated Systems".

\section{REFERENCES}

[1] I. Kore et al., "Multi-site test of RF transceivers on low-cost digital ATE," Proc. IEEE International Test Conference (ITC), pp. 1-10, 2011.

[2] C. H. Peng et al., "A novel RF self test for a combo SoC on digital ATE with multi-site applications," Proc. Int'1 Test Conf. (ITC), p. 8, 2014.

[3] M. Ishida K. Ichiyama, "An ATE System for Testing RF Digital Communication Devices With QAM Signal Interfaces," IEEE Design \& Test, vol. 33, no. 6, pp. 15-22, 2016.

[4] N. Pous et al., J.,"A Level-Crossing Approach for the Analysis of RF Modulated Signals using only Digital Test Resources", J. of Electronic Testing: Theory and App. (JETTA), vol. 27, no. 3, pp 289-303, 2011

[5] S. David-Grignot et al., "Low-cost phase noise testing of complex RF ICs using standard digital ATE," Proc. IEEE Int'l Test Conf. (ITC), p. 9, 2014.

[6] J-L Huang, K-T Cheng, "An on-chip short-time interval measurement technique for testing high-speed communication links," Proc. IEEE VLSI Test Symposium (VTS), pp. 380-385, 2001.

[7] S. Sunter, A. Roy, "On-chip digital jitter measurement, from megahertz to gigahertz," IEEE Design \& Test of Computers, vol. 21, no. 4, pp. 314-321, 2004.

[8] T. Vayssade et al., "Low-cost digital test solution for symbol error detection of RF ZigBee transmitters", IEEE Trans. On Device \& Materials Reliability, vol. 19, no. 1, pp. 16-24, 2019.

[9] T. Vayssade et al., "Power measurement and spectral test of ZigBee transmitters from 1-bit under-sampled acquisition", Proc. IEEE European Test Symposium (ETS), p.6, 2019. 